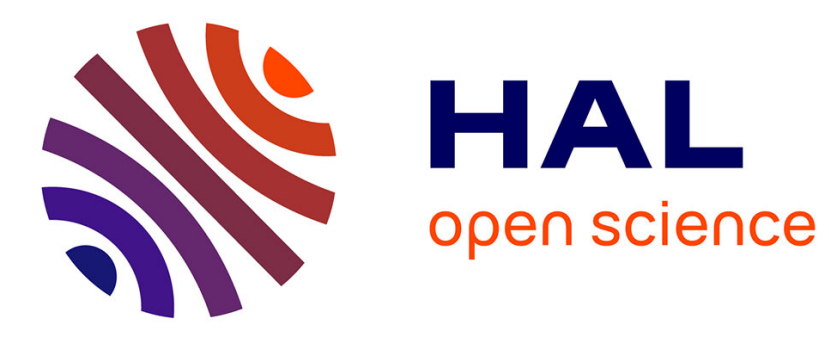

\title{
Coping with Semilattices of Relations in Logics with Relative Accessibility Relations
}

\author{
Stéphane Demri
}

\section{To cite this version:}

Stéphane Demri. Coping with Semilattices of Relations in Logics with Relative Accessibility Relations. Ewa Orlowska; Andrzej Szalas. Relational Methods for Computer Science Applications. Selected Papers from 4th International Seminar on Relational Methods in Logic, Algebra and Computer Science (RelMiCS'98), , pp.163-181, 2001, 978-3-7908-1828-4. 10.1007/978-3-7908-1828-4_10 . hal-03194796

\section{HAL Id: hal-03194796 https://hal.science/hal-03194796}

Submitted on 9 Apr 2021

HAL is a multi-disciplinary open access archive for the deposit and dissemination of scientific research documents, whether they are published or not. The documents may come from teaching and research institutions in France or abroad, or from public or private research centers.
L'archive ouverte pluridisciplinaire HAL, est destinée au dépôt et à la diffusion de documents scientifiques de niveau recherche, publiés ou non, émanant des établissements d'enseignement et de recherche français ou étrangers, des laboratoires publics ou privés. 


\title{
Coping with Semilattices of Relations in Logics with Relative Accessibility Relations
}

\author{
Stéphane Demri \\ Laboratoire LEIBNIZ - C.N.R.S. \\ 46 Avenue Félix Viallet \\ 38031 Grenoble, France \\ email: demri@imag.fr
}

December 17, 2004

\begin{abstract}
We present a class of polymodal logics for which the set of terms indexing the modal connectives can be hierarchized in two levels: the set of Boolean terms and the set of terms built upon the set of Boolean terms. The semantical structures of the logics contains a family of binary relations that can be viewed as an homomorphism between semilattices. Various results related to decidability, axiomatization and computational complexity are established by faithfully translating the logics into more standard modal logics. The paper is a short survey of results obtained by translation for various logics of the above kind from the literature.
\end{abstract}

Keywords: modal logic, relative operator, reduction

\section{Introduction}

The information logics derived from Pawlak's information systems [Paw81] are polymodal logics such that the relations in the models correspond to relations derived from information systems. Let us recall the peculiarities of such logics. First, numerous propositional modal logics can be defined from semantical structures of the form $\mathcal{M}=\left\langle W,\left(R_{\mathrm{a}}\right)_{\mathrm{a} \in \mathbb{M}}, V\right\rangle$ where $W$ is a non-empty set, $\left(R_{\mathrm{a}}\right)_{\mathrm{a} \in \mathbb{M}}$ is a family of binary relations over $W, \mathrm{M}$ is the set of modal expressions and $V$ is a meaning function that is, for each propositional variable $\mathrm{p}$, 
$V(\mathrm{p}) \subseteq W$. The well-known propositional dynamic logic PDL (see e.g. [Har84]) uses this kind of semantical structures. In the sequel, such logics are called standard modal logics. By adding a Boolean dimension to the standard modal logics we obtain a class of logics (the logics with Relative Accessibility RElations or shorter, the "Rare-logics") that includes numerous information logics (see [Orło84a, Orło84b]). At the syntactical level, we replace the set of modal constants (upon which is built the set $\mathrm{M}$ of modal expressions) by a set of expressions of the form $r(\alpha)$ with $\alpha \in \mathrm{P}$ where the set $\mathrm{P}$ is built upon a set $\mathrm{P}_{0}=\left\{\delta_{i}: i \in \omega\right\}$ of parameter constants and is closed under the Boolean operators $\cap, \cup,-$. The syntactic construction $r($.$) announces$ a parameter expression. The semantical structures are of the form $\left\langle W, P A R,\left(\mathcal{R}_{P}\right)_{P \subseteq P A R}, V\right\rangle$ where $P A R$ is a non-empty set of parameters, $V$ also maps homomorphically every Boolean expression $\alpha$ to a subset of $P A R$ and $V(r(\alpha))=\mathcal{R}_{V(\alpha)}$ for any $\alpha \in$ P. According to the algebraic properties of $\left(\mathcal{R}_{P}\right)_{P \subseteq P A R}$, different types of logics are defined (see e.g. [Orło89, Bal97, Kon97]).

The main result of the paper states that there exist satisfiabilitypreserving transformations between Rare-logics and the corresponding standard modal logics (validity-preserving maps can be also designed). The translations are interesting for their own sake, for instance they help understanding what is brought by adding a Boolean dimension to a logic. By taking advantage of such translations, we provide a uniform framework to prove decidability for various Rare-logics from [Orło84a, Orło89, Orło88, Bal96a, Bal97]. Moreover, we show how it can also help to define proof systems for the Rare-logics. Quantitative aspects about computational complexity are also briefly discussed.

As far as we know, the notion of Rare-logic appeared in the literature in [Orło88] where various Rare-logics are described to model reasoning in the presence of incomplete information. Hilbert-style and Rasiowa-Sikorski-style calculi for some Rare-logics have been defined in [Bal96a, Bal97, Kon97]. Recently, in [BO99], a classification of logics with relative accessibility relations has been proposed. None of these works tackle the problem of relating Rare-logics with more standard modal logics in a systematic way. Moreover, in these works, decidability issues are not their concern. Decidability of information logics has been established in the past by using filtration-like and restrictionlike techniques (see e.g. [Vak91, Dem98a]). Herein, we use a different approach that interprets a Rare-logic into a standard modal logic fol- 
lowing the general framework established from [Tar53].

The paper is organised as follows. In Section 2, we define the class of standard modal logics as well as the class of Rare-logics considered in the paper. In Section 3, we define general properties about semilattices and then show how they can be used to define satisfiability-preserving maps between Rare-logics and standard modal logics. In Section 4, we establish various decidability results whereas in Section 5 we provide a sufficient condition to prove that the satisfiability problem of a Rarelogic is in the complexity class EXPTIME (that is, it can be solved by a deterministic Turing machine in exponential-time with respect to the size of the input formulae). In Section 6, we propose a general recipe to define proof systems for Rare-logics that is illustrated on a particular example. We round off the paper by open questions that are worth being investigated.

This paper is a full version of the extended abstract [Dem98b]. Most of the results of this paper can be extended to the case when nominals are allowed in the language (see [DG00a, DK98]) or to the case when the accessibility relations of the semantical structures can be of arbitrary arity (see also [DG00a]). Some other related works can be found in [DG00a, Dem99, DG00b].

\section{Modal logics}

\section{$2.1 \quad$ Language}

Let $O P=\left\{\oplus_{1}, \ldots, \oplus_{s}\right\}$ be a (possibly empty) finite set of operators such that $\rho\left(\oplus_{i}\right) \in \omega \backslash\{0\}$ denotes the arity of $\oplus_{i}$. A set $\mathrm{M}=\{\mathrm{a}, \mathrm{b}, \ldots\}$ of modal expressions is the smallest set that contains a non-empty countable set $\mathrm{M}_{0}$ of basic modal expressions and it is closed under $O P$ with respect to the arity map $\rho$. A modal language $\mathrm{L}$ is defined as a pair $\left\langle\left\langle O P, \rho, \mathrm{M}_{0}, \mathrm{M}\right\rangle, \mathrm{For}_{0}\right\rangle$ where $\mathrm{For}_{0}=\{\mathrm{p}, \mathrm{q}, \ldots\}$ is a countable set of propositional variables. The formulae $\phi$ of $\mathrm{L}$ are inductively defined as follows: $\phi::=\mathrm{p}\left|\phi_{1} \wedge \phi_{2}\right| \neg \phi \mid[\mathrm{a}] \phi$ for $\mathrm{p} \in$ For $_{0}$ and $\mathrm{a} \in \mathrm{M}$. The set of L-formulae is denoted For. Standard abbreviations include $\perp$, $\top,\langle\mathrm{a}\rangle, \vee, \Rightarrow, \Leftrightarrow$. We write $\operatorname{sub}(\phi)$ to denote the set of subformulae of the formula $\phi$. 


\section{$2.2 \quad$ Kripke-style semantics}

A relation operation $g$ maps any set $U$ to a mapping $g(U): \mathcal{P}\left(U^{i_{1}}\right) \times$ $\ldots \times \mathcal{P}\left(U^{i_{n}}\right) \rightarrow \mathcal{P}\left(U^{i_{n+1}}\right)$ with $i_{1}, \ldots, i_{n+1} \geq 1$ and $i_{1}, \ldots, i_{n+1}$ do not depend on $U$. $\left\langle i_{1}, \ldots, i_{n+1}\right\rangle$ is the profile of $g$ and $n$ is its arity. We also require that if there is an 1-1 mapping $f: U \rightarrow U^{\prime}$, then for any $\left\langle X_{1}, \ldots X_{n}\right\rangle \in \mathcal{P}\left(U^{i_{1}}\right) \times \ldots \times \mathcal{P}\left(U^{i_{n}}\right)$,

$$
\left.f\left(g(U)\left(X_{1}, \ldots, X_{n}\right)\right)=g\left(U^{\prime}\right)\left(f\left(X_{1}\right), \ldots, f\left(X_{n}\right)\right)\right)
$$

An operator interpretation $\mathcal{I}$ maps the set $O P$ into the set of relation operations such that for any $\oplus \in O P$ such that $\rho(\oplus)=n$, the profile of $\mathcal{I}(\oplus)$ is $\langle 2, \ldots, 2,2\rangle$, an $n+1$-tuple. A similar definition is used in [DO99]. An L-frame $\mathcal{F}$ is a pair $\left\langle W,\left(R_{\mathrm{a}}\right)_{\mathrm{a} \in \mathrm{M}}\right\rangle$ such that $W$ is a non-empty set and for any a $\in \mathrm{M}, R_{\mathrm{a}} \subseteq W^{2}$. An L-model $\mathcal{M}$ respecting some operator interpretation $\mathcal{I}$ is a structure $\mathcal{M}=\left\langle W,\left(R_{\mathrm{a}}\right)_{\mathrm{a} \in \mathrm{M}}, V\right\rangle$ such that $\left\langle W,\left(R_{\mathrm{a}}\right)_{\mathrm{a} \in \mathrm{M}}\right\rangle$ is an L-frame, $V$ is a map $V:$ For $_{0} \rightarrow \mathcal{P}(W)$ and for any $\oplus\left(\mathrm{a}_{1}, \ldots, \mathrm{a}_{n}\right) \in \mathrm{M}, R_{\oplus\left(\mathrm{a}_{1}, \ldots, \mathrm{a}_{n}\right)}=\mathcal{I}(\oplus)(W)\left(R_{\mathrm{a}_{1}}, \ldots, R_{\mathrm{a}_{n}}\right)$. The formula $\phi$ is satisfied by the world $u \in W$ in $\mathcal{M}$ iff $\mathcal{M}, u \models \phi$ where the satisfaction relation $\models$ is inductively defined as follows:

1. $\mathcal{M}, u \models \mathrm{p} \stackrel{\text { def }}{\Leftrightarrow} u \in V(\mathrm{p})$, for any $\mathrm{p} \in \mathrm{For}_{0}$;

2. $\mathcal{M}, u \models \neg \phi \stackrel{\text { def }}{\Leftrightarrow} \operatorname{not} \mathcal{M}, u \models \phi$;

3. $\mathcal{M}, u \models \phi \wedge \psi \stackrel{\text { def }}{\Leftrightarrow} \mathcal{M}, u \models \phi$ and $\mathcal{M}, u \models \psi$;

4. $\mathcal{M}, u \models[\mathrm{a}] \phi \stackrel{\text { def }}{\Leftrightarrow}$ for all $u^{\prime} \in R_{\mathrm{a}}(u), \mathcal{M}, u^{\prime} \models \phi$.

A formula $\phi$ is true in an L-model $\mathcal{M}$ (denoted by $\mathcal{M}=\phi) \stackrel{\text { def }}{\Leftrightarrow}$ for any $u \in W, \mathcal{M}, u \models \phi . \phi$ is valid in an L-frame $\mathcal{F}$ (denoted by $\mathcal{F} \models \phi$ ) $\stackrel{\text { def }}{\Leftrightarrow} \mathcal{M} \models \phi$ for any L-model based on $\mathcal{F}$.

\subsection{Classes of logics}

An L-normal modal logic $\mathcal{L}$ is defined as a triple $\langle\mathrm{L}, \mathcal{I}, \mathcal{C}\rangle$ such that $\mathcal{I}$ is an operator interpretation and $\mathcal{C}$ is a class of L-models respecting $\mathcal{I}$. A standard modal logic is an L-normal modal logic such that the set of basic modal expressions is a countable (possibly finite) set $\mathrm{M}_{0}=$ $\left\{c_{0}, c_{1}, \ldots\right\}$ of constants. An L-formula $\phi$ is said to be $\mathcal{L}$-valid iff $\phi$ is true in all the $\mathcal{L}$-models. An L-formula $\phi$ is said to be $\mathcal{L}$-satisfiable iff there exist an $\mathcal{L}$-model $\mathcal{M}=\left\langle W,\left(R_{\mathrm{a}}\right)_{\mathrm{a} \in \mathrm{M}}, V\right\rangle$ and $u \in W$ such that $\mathcal{M}, u \models \phi$. We say that $\psi$ is a logical $\mathcal{L}$-consequence of $\phi$ (in symbols 
$\phi \models \mathcal{L} \psi) \stackrel{\text { def }}{\Leftrightarrow}$ for any $\mathcal{L}$-model $\mathcal{M}, \mathcal{M} \models \phi$ implies $\mathcal{M}=\psi$. As usual, we say that the modal logic $\mathcal{L}$ has the finite model property (fmp) iff each $\mathcal{L}$-satisfiable formula $\phi$ has an $\mathcal{L}$-model of finite cardinality.

A set of modal expressions is said to be designed for Rare-logics if each basic modal expression is of the form $r(\alpha)$ where $\alpha$ is a parameter expression and ' $r$ ' is an arbitrary symbol fixed in the rest of the paper. The set $\mathrm{P}=\{\alpha, \beta, \ldots\}$ of parameter expressions is the smallest set containing a countable set $\mathrm{P}_{0}=\left\{\delta_{i}: i \in \omega\right\}$ of parameter constants and it is closed under the Boolean operators $\cap, \cup,-$. A P-valuation $V$ is a map $V: \mathrm{P} \rightarrow \mathcal{P}(P A R)$ such that for any $\alpha_{1}, \alpha_{2} \in \mathrm{P}$,

- $V\left(\alpha_{1}\right) \in \mathcal{P}(P A R) ; V\left(-\alpha_{1}\right)=P A R \backslash V\left(\alpha_{1}\right)$;

- $V\left(\alpha_{1} \cap \alpha_{2}\right)=V\left(\alpha_{1}\right) \cap V\left(\alpha_{2}\right) ; V\left(\alpha_{1} \cup \alpha_{2}\right)=V\left(\alpha_{1}\right) \cup V\left(\alpha_{2}\right)$.

For any $\alpha, \beta \in \mathrm{P}$ we write $\alpha \equiv \perp[$ resp. $\alpha \equiv \beta, \alpha \sqsubseteq \beta$ ] when for any P-valuation $V, V(\alpha)=\emptyset$ [resp. $V(\alpha)=V(\beta), V(\alpha) \subseteq V(\beta)]$. The relations $\equiv$ and $\sqsubseteq$ are known to be decidable (by decidability of classical propositional logic for instance). Let L be a modal language designed for Rare-logics. An L-model $\mathcal{M}=\left\langle W,\left(R_{\mathrm{a}}\right)_{\mathrm{a} \in \mathrm{M}}, V\right\rangle$ is said to be designed for Rare-logics $\stackrel{\text { def }}{\Leftrightarrow}$ there exist a non empty set $P A R$, a family $\left(\mathcal{R}_{P}\right)_{P \subseteq P A R}$ of binary relations over $W$ and a P-valuation $V^{\prime}$ such that for any $\alpha \in \mathrm{P}, \mathcal{R}_{V^{\prime}(\alpha)}=R_{r(\alpha)}$. Such a model $\mathcal{M}$ is denoted $\left\langle W, P A R,\left(\mathcal{R}_{P}\right)_{P \subseteq P A R}, V^{\prime \prime}\right\rangle$ where $V^{\prime \prime}$ is a map $V^{\prime \prime}:\left(\right.$ For $\left._{0} \cup \mathrm{P} \cup \mathrm{M}\right) \rightarrow$ $\mathcal{P}(P A R) \cup \mathcal{P}(W) \cup \mathcal{P}\left(W^{2}\right)$ such that the restriction of $V^{\prime \prime}$ to $\mathrm{P}$ is $V^{\prime}$, the restriction of $V^{\prime \prime}$ to For $_{0}$ is $V$ and for any a $\in \mathrm{M}, V^{\prime \prime}(\mathrm{a})=R_{\mathrm{a}}$.

We write $F r^{2}$ to denote the class of structures $\langle W, R\rangle$ where $W$ is a non-empty set and $R \subseteq W^{2}$.

Definition 2.1. Let L be a modal language designed for Rare-logics. An L-normal modal logic $\mathcal{L}=\langle\mathrm{L}, \mathcal{I}, \mathcal{C}\rangle$ is said to be a Rare-logic iff there is a class $X \subseteq F r^{2}$ such that $\mathcal{C}$ is the class of L-models $\mathcal{M}=$ $\left\langle W, P A R,\left(\mathcal{R}_{P}\right)_{P \subseteq P A R}, V\right\rangle$ respecting $\mathcal{I}$ satisfying for any $\emptyset \neq P \subseteq$ $P A R,\left\langle W, \mathcal{R}_{P}\right\rangle \in X$.

$\mathcal{L}$ is also written $\langle\mathrm{L}, \mathcal{I}, \mathcal{C}, X\rangle$. What mainly motivates the introduction of the class of Rare-logics can be found below. During the last decade, the information logics derived from Pawlak's information systems [Paw81] have been the object of quite active research. An information system can be seen as a structure $\langle O B, A T\rangle$ such that $O B$ is a non-empty set of objects, $A T$ is a non-empty set of attributes and 
each attribute at $\in A T$, is a mapping at $: O B \rightarrow \mathcal{P}\left(V_{a l} l_{a t}\right)$ where $V_{a l}$ is a non-empty set of values. For each object $o$ and for each attribute at, at $(o)$ is the set of possible values of $o$ with respect to the attribute at. This is at least one possible reading for at $(o)$ (one can think also about its conjunctive reading). Usually, at (o) is assumed to be non-empty. Such structures are intended to capture some aspects of incomplete information. In that setting, various derived relations between objects can be defined. We recall below the indiscernibility and positive similarity relations. For any $o_{1}, o_{2} \in O B, A \subseteq A T$,

- $o_{1} \operatorname{ind}(A) o_{2} \stackrel{\text { def }}{\Leftrightarrow}$ for any at $\in A$, at $\left(o_{1}\right)=a t\left(o_{2}\right)$;

- $o_{1} \operatorname{sim}(A) o_{2} \stackrel{\text { def }}{\Leftrightarrow}$ for any at $\in A$, at $\left(o_{1}\right) \cap a t\left(o_{2}\right) \neq \emptyset$.

$o_{1}$ ind $(A) o_{2}$ can be read as follows: the objects $o_{1}$ and $o_{2}$ cannot be distinguished modulo the set of attributes $A$. Similarly, $o_{1} \operatorname{sim}(A) o_{2}$ iff $o_{1}$ and $o_{2}$ are similar modulo $A$. The polymodal logics obtained from the information systems are multimodal logics such that the relations in the Kripke-style semantical structures correspond to relations between objects in the underlying information systems. Usually, such logics are supposed to perform reasoning about information systems. It is not clear to us that those logics satisfy their initial goal however they offer other remarkable properties. For instance, the accessibility relations in the models are interdependent; for instance, if $B \subseteq A \subseteq A T$, then $\operatorname{ind}(A) \subseteq \operatorname{ind}(B)$. Moreover, the set of modal terms can be hierarchized in two levels: the set of Boolean terms (interpreted as "sets of attributes") and the set of modal expressions (interpreted as "derived relations from information systems").

The main purpose of this work is to associate a standard modal logic to each Rare-logic in order to study the Rare-logic via properties of the standard modal logic. In general, the standard modal logics and the Rare-logics share neither the same type of modal language nor the same type of semantical structures. However, the main nontechnical difference between these logics can be found in the following observation. Two kinds of syntactic objects can be distinguished for standard modal logics: the modal expressions (interpreted as binary relations) and the formulas (interpreted as sets of worlds). In the Rarelogics, a third kind of syntactic objects is introduced: the parameter expression (interpreted as sets of attributes). This third component introduces new constraints on the semantical structures and this is our intention in this paper to show why this seemingly increase of 
complexity is sometime only superficial.

Definition 2.2. Let $\mathcal{L}=\langle\mathrm{L}, \mathcal{I}, \mathcal{C}, X\rangle$ be a Rare-logic. The standard modal $\operatorname{logic} \mathcal{L}_{d}$ from $\mathcal{L}$ is the structure $\left\langle\mathrm{L}_{d}, \mathcal{I}_{d}, \mathcal{C}_{d}\right\rangle$ such that

- $\mathrm{L}_{d}$ is the modal language obtained from $\mathrm{L}$ by replacing $\{r(\alpha)$ : $\alpha \in \mathrm{P}\}$ by the set $\left\{\mathrm{c}_{i}: i \in \omega\right\}$ of modal constants;

- $\mathcal{I}_{d}=\mathcal{I}$

- for any $\mathrm{L}_{d}$-model $\mathcal{M}=\left\langle W,\left(R_{\mathrm{a}}\right)_{\mathrm{a} \in \mathrm{M}}, V\right\rangle, \mathcal{M} \in \mathcal{C}_{d} \stackrel{\text { def }}{\Leftrightarrow} \mathcal{M}$ respects $\mathcal{I}$, and the relations generated from $\left\{R_{\mathrm{C}}: \mathrm{c} \in \mathrm{M}_{0}\right\}$ with intersection belong to $X$.

In [DG00a, Part I], the definition of the standard modal logic from a Rare-logic is more involved and more general. Indeed, in the present paper, we only provide developments for a single (forthcoming) class of Rare-logics. In this work, we shall consider algebras $\langle D, \sqcap\rangle$ of type $\langle 2\rangle$ such that $\square$ is commutative, associative, idempotent with a zero element $e$. In particular, any join-semilattice [resp. meet-semilattice] $\langle D, \leq\rangle$ with a bottom element $\perp$ [resp. with a top element $T$ ], can be seen as an algebra of that kind by defining for any $a, b \in D, a \sqcap b \stackrel{\text { def }}{=} a \vee b$ [resp. $a \sqcap b \stackrel{\text { def }}{=} a \wedge b$ ]. Similarly, any algebra $\langle D, \sqcap\rangle$ of the above kind can be seen for instance as a join-semilattice by defining for any $a, b \in D$, $a \leq b \stackrel{\text { def }}{\Leftrightarrow} a \sqcap b=b$. By abusing our notation, we write $\langle D, \sqcap, e\rangle$ to denote the algebras of the above class and we call them semilattices with zero element e. Indeed, we shall mainly investigate Rare-logics for which the sets of relations of the models are semilattices with zero element. Indeed, we consider the case when the family $\left(\mathcal{R}_{P}\right)_{P \subseteq P A R}$ is exactly a map $g:\langle\mathcal{P}(P A R), \cup, \emptyset\rangle \rightarrow\left\langle\mathcal{P}\left(W^{2}\right), \sqcap, W^{2}\right\rangle$ where

1. $\langle\mathcal{P}(P A R), \cup, \emptyset\rangle$ is the semilattice where $\cup$ is the set union;

2. $\left\langle\mathcal{P}\left(W^{2}\right), \sqcap, W^{2}\right\rangle$ is a semilattice with zero element $W^{2}$;

3. $g(\emptyset)=W^{2}$;

4. $g$ is an homomorphism, that is for any $P, P^{\prime} \in \mathcal{P}(P A R), g(P \cup$ $\left.P^{\prime}\right)=g(P) \sqcap g\left(P^{\prime}\right)$;

5. for any $P \subseteq P A R, \mathcal{R}_{P}=g(P)$. 
In [Nov97, Jär97] algebraic structures similar to semilattices have been investigated in order to study the dependence spaces and information systems. Other conditions on the family $\left(\mathcal{R}_{P}\right)_{P \subseteq P A R}$ exist in the literature (see e.g. [Dün97, Orło93, Kon97, Bal97]). We concentrate here on Rare-logics $\mathcal{L}=\langle\mathrm{L}, \mathcal{I}, \mathcal{C}, X\rangle$ where there is an operator $\oplus_{\mathcal{L}} \in O P$ such that for any L-model $\mathcal{M}=\left\langle W, P A R,\left(\mathcal{R}_{P}\right)_{P \subseteq P A R}, V\right\rangle, \mathcal{M} \in \mathcal{C}$ $\stackrel{\text { def }}{\Leftrightarrow}$

1. $\mathcal{M}$ respects $\mathcal{I}$ and for any $\emptyset \neq P \subseteq P A R,\left\langle W, \mathcal{R}_{P}\right\rangle \in X$;

2. $\mathcal{I}\left(\oplus_{\mathcal{L}}\right)(W)=\sqcap$;

3. for any $P, P^{\prime} \subseteq P A R, \mathcal{R}_{P \cup P^{\prime}}=\mathcal{R}_{P} \sqcap \mathcal{R}_{P^{\prime}}$;

4. $\mathcal{R}_{\emptyset}=W^{2}$.

As a consequence for such Rare-logics, the class $X$ of modal frames is closed under $\sqcap$. The technical developments are done in this paper for these kinds of Rare-logics. Other classes are treated in [DG00a, Part I].

Example 2.1. Let $L I R=\langle\mathrm{L}, \mathcal{I}, \mathcal{C}, X\rangle$ be the Rare-logic such that $O P=\left\{\cap, \cup^{*}\right\}$ (respectively interpreted as the intersection and the reflexive and transitive closure of the union on binary relations), $X$ is the class of equivalence relations and $\sqcap$ is interpreted as the set intersection. The corresponding standard modal logic $L I R_{d}$ is precisely the logic DAL defined in [FdCO85]. DAL is a standard (poly)modal logic where the modal terms are built upon the modal constants $\left\{\mathrm{c}_{i}: i \in \omega\right\}$ and closed under $\cap$ and $\cup^{*}$. Furthermore, each $c_{i}$ is interpreted as an equivalence relation.

Let $\mathcal{L}_{d}=\left\langle\mathrm{L}_{d}, \mathcal{I}_{d}, \mathcal{C}_{d}\right\rangle$ be a standard modal logic. We write $\mathcal{L}_{d}^{\mathrm{U}}$ to denote the modal logic obtained from $\mathcal{L}_{d}$ by adding the nullary modal operator $\mathrm{U}$ and the class of models is obtained from $\mathcal{C}_{d}$ by imposing that $R_{\mathrm{U}}=W^{2}$ (see e.g. [GP92, Hem96]). We denote by $\mathcal{L}_{d}^{\mathrm{U}^{-}}$the logic obtained from $\mathcal{L}_{d}^{\mathrm{U}}$ by only allowing the occurrences of $\mathrm{U}$ of the form $[\mathrm{U}] . \quad \mathcal{L}_{d}^{\mathrm{U}}$ is said to be $\mathrm{U}$-simplifiable iff there is an effective procedure $f:$ For $\rightarrow$ For such that for any $\phi \in$ For: the only occurrences of $\mathrm{U}$ in $f(\phi)$ are in the context [U] and $f(\phi) \Leftrightarrow \phi$ is $\mathcal{L}_{d}^{\mathrm{U}}$-valid. For instance, for the standard modal logic DAL -see Example $2.1-\mathrm{DAL}^{\mathrm{U}}$ is U-simplifiable. 


\section{$3 \quad$ Satisfiability-preserving maps}

\subsection{Constructions on semilattices}

Let $\mathrm{L}$ be a modal language designed for Rare-logics and let $\mathrm{M}_{0 d}=$ $\left\{\mathrm{c}_{i}: i \in \omega\right\}$ be a countably infinite set of modal constants (intended to belong to the language of a standard modal logic). Let $\delta_{1}, \ldots, \delta_{n}$ be elements of $\mathrm{P}_{0}$. For any integer $k \in\left\{0, \ldots, 2^{n}-1\right\}$, we write $\alpha_{k}^{*}$ to denote the Boolean expression (also called a component) $\alpha_{k}^{*} \stackrel{\text { def }}{=}$ $\alpha_{1} \cap \ldots \cap \alpha_{n}$ where for any $s \in\{1, \ldots, n\}, \alpha_{s} \stackrel{\text { def }}{=} \delta_{s}$ if bit $t_{s}(k)=0\left(b_{i t}(k)\right.$ denoting the $s$ th bit in the binary representation of $k$ ) otherwise $\alpha_{s} \stackrel{\text { def }}{=}$ $-\delta_{s}$. For instance,

$$
\alpha_{2^{n-1}-1}^{*}=-\delta_{1} \cap \ldots \cap-\delta_{n-1} \cap \delta_{n}
$$

For any P-valuation $V,\left\{V\left(\alpha_{k}^{*}\right): k \in\left\{0, \ldots, 2^{n}-1\right\}\right\}$ is a partition of $P A R$. For any parameter expression $\alpha \in \mathrm{P}$ such that the only parameter constants occurring in $\alpha$ are in $\left\{\delta_{1}, \ldots, \delta_{n}\right\}$, either $\alpha \equiv \perp$ or there is a unique non-empty set $Y=\left\{\alpha_{i_{1}}^{*}, \ldots, \alpha_{i_{l}}^{*}\right\}$ such that $\alpha \equiv$ $\alpha_{i_{1}}^{*} \cup \ldots \cup \alpha_{i_{1}}^{*}$. There exists an effective procedure that computes $Y$ in deterministic exponential-time in the size of $\alpha$. Proposition 3.1 below states how to transform a family $\left(X_{P}\right)_{P \subseteq P A R}$ into a family $\left(Y_{\mathrm{C}}\right)_{\mathrm{c} \in \mathrm{M}_{0 d}}$ when both families can be seen as the carrier sets of semilattices.

Proposition 3.1. Let $\delta_{1}, \ldots, \delta_{n}$ be $n>0$ elements of $\mathrm{P}_{0},\left\langle\left\{X_{P}\right.\right.$ : $P \subseteq P A R\}, \sqcap, e\rangle$ be a semilattice with zero element $e$ and $V$ be a P-valuation $V: \mathrm{P} \rightarrow \mathcal{P}(P A R)$ such that (H1) $X_{\emptyset}=e$ and (H2) for any $P, P^{\prime} \subseteq P A R, X_{P \cup P^{\prime}}=X_{P} \sqcap X_{P^{\prime}}$. Then, there is a family $\left(Y_{\mathrm{C}}\right)_{\mathrm{c} \in \mathrm{M}_{0 d}}$ such that

(C1) $\left\{Y_{\mathrm{C}}: \mathrm{c} \in \mathrm{M}_{0 d}\right\}$ is a finite subset of $\left\{X_{P}: P \subseteq P A R\right\}$;

(C2) If $\alpha$ is a parameter expression built from the parameter constants $\delta_{1}, \ldots, \delta_{n}$ such that $\alpha \equiv \alpha_{i_{1}}^{*} \cup \ldots \cup \alpha_{i_{l}}^{*}$, then $X_{V(\alpha)}=Y_{\mathrm{C}_{i_{1}}} \sqcap \ldots \sqcap$ $Y_{\mathrm{C}_{i_{l}}}$.

Proposition 3.2 below states how to transform a family $\left(Y_{\mathbf{C}}\right)_{\mathbf{C} \in \mathbb{M}_{0 d}}$ into a family $\left(X_{P}\right)_{P \subseteq P A R}$ when both families can be seen as the carrier set of semilattices with zero element.

Proposition 3.2. Let $\delta_{1}, \ldots, \delta_{n}$ be $n>0$ elements of $\mathrm{P}_{0},\langle Y, \sqcap, e\rangle$ be a semilattice with zero element and $\left(Y_{\mathrm{C}}\right)_{\mathrm{C} \in \mathrm{M}_{0 d}}$ be an indexed family 
of elements of $Y$. Then, there is a subalgebra of $\langle Y, \sqcap, e\rangle$ of the form $\left\langle\left\{X_{P}: P \subseteq P A R\right\}, \sqcap, e\right\rangle$ satisfying the conditions (H1)-(H2) from Proposition 3.1 and there is a P-valuation $V: \mathrm{P} \rightarrow \mathcal{P}(P A R)$ such that

(C1) $\operatorname{card}(P A R)=2^{n}$;

(C2) If $\alpha$ is a parameter expression built from the parameter constants $\delta_{1}, \ldots, \delta_{n}$ such that $\alpha \equiv \alpha_{i_{1}}^{*} \cup \ldots \cup \alpha_{i_{l}}^{*}$, then $X_{V(\alpha)}=Y_{\mathrm{C}_{i_{1}}} \sqcap \ldots \sqcap$ $Y_{\mathrm{C}_{i_{l}}}$.

Proof: Let $\left\langle\left\langle\left\{X_{P}: P \subseteq P A R\right\}, \sqcap, e\right\rangle, V\right\rangle$ be the structure defined as follows:

- $P A R \stackrel{\text { def }}{=}\left\{0, \ldots, 2^{n}-1\right\}$;

- $X_{\emptyset} \stackrel{\text { def }}{=} e$ and for any $\emptyset \neq P \subseteq P A R, X_{P} \stackrel{\text { def }}{=} \sqcap_{k \in P} Y_{\mathrm{C}_{k}}$ ( $\sqcap$ is commutative, associative and each $P$ is finite);

- for any $s \in\{1, \ldots, n\}, V\left(\delta_{s}\right) \stackrel{\text { def }}{=}\left\{k \in P A R: b i t_{s}(k)=0\right\}$ (for the other parameter constants $V$ is not constrained until $V$ is a $\mathrm{P}$-valuation which is always possible).

$\left\langle\left\langle\left\{X_{P}: P \subseteq P A R_{i}\right\}, e, \sqcap\right\rangle, V\right\rangle$ satisfies the required conditions. By way of example, let us check that the condition (C2) holds. First, observe that for any $k \in\left\{0, \ldots, 2^{n}-1\right\}, V\left(\alpha_{k}^{*}\right)=\{k\}$.

$$
\begin{aligned}
X_{V(\alpha)} & =X_{V\left(\alpha_{i_{1}}^{*} \cup \ldots \cup \alpha_{i_{l}}^{*}\right)}(\text { normal form of } \alpha) \\
& =X_{V\left(\alpha_{i_{1}}^{*}\right) \cup \ldots \cup V\left(\alpha_{i_{l}}^{*}\right)}(V \text { is a P-valuation) } \\
& =X_{V\left(\alpha_{i_{1}}^{*}\right)} \sqcap \ldots \sqcap X_{V\left(\alpha_{i_{l}}^{*}\right)} \text { (by (H2)) } \\
& =X_{\left\{i_{1}\right\}} \sqcap \ldots \sqcap X_{\left\{i_{l}\right\}} \\
& =Y_{\mathrm{C}_{i_{1}}} \sqcap \ldots \sqcap Y_{\mathrm{C}_{i_{l}}} \text { (by construction) }
\end{aligned}
$$

\section{Q.E.D.}

In Proposition 3.1 and in Proposition 3.2 the $X_{P}$ 's and $Y_{\mathrm{C}}$ 's are not necessarily relations. We are going to take advantage of these propositions when dealing with possible-world semantics for polymodal modal logics.

\subsection{Normalization}

Let $\phi$ be an L-formula such that $\mathrm{P}_{0}(\phi) \subseteq\left\{\delta_{1}, \ldots, \delta_{n}\right\}$ where $\mathrm{P}_{0}(\phi)$ is the set of parameter constants occurring in $\phi$ (the case when $\mathrm{P}_{0}(\phi)=\emptyset$ is omitted herein but it poses no extra difficulties). The first normal form 
of $r(\alpha)$, written $\mathrm{N}_{1}(r(\alpha))$, is the basic modal expression $r\left(\alpha_{i_{1}}^{*} \cup \ldots \cup \alpha_{i_{l}}^{*}\right)$. It is similar to the canonical disjunctive normal form for the propositional calculus. In the case when $\alpha \equiv \perp, \mathrm{N}_{1}(r(\alpha)) \stackrel{\text { def }}{=} r\left(\delta_{1} \cap-\delta_{1}\right)$. We write $\mathrm{N}_{1}(\phi)$ to denote the formula obtained from $\phi$ by substituting each occurrence of $r(\alpha)$ by $\mathrm{N}_{1}(r(\alpha)) . \mathrm{N}_{1}(\phi)$ is unique modulo associativity and commutativity of $\cup$ and $\cap$ (which is harmless in the sequel). The technique of components has been firstly used for information logics by B. Konikowska (see e.g. [Kon97]) in order to define Rasiowa-Sikorski proof systems for relative similarity logics. The second normal form of $\phi$, written $\mathrm{N}_{2}(\phi)$, is the formula obtained from $\mathrm{N}_{1}(\phi)$ where each occurrence of $r\left(\alpha_{i_{1}}^{*} \cup \ldots \cup \alpha_{i_{l}}^{*}\right)$ has been substituted by $r\left(\alpha_{i_{1}}^{*}\right) \oplus_{\mathcal{L}} \ldots \oplus_{\mathcal{L}} r\left(\alpha_{i_{l}}^{*}\right)$. Obviously, $\phi \Leftrightarrow \mathrm{N}_{2}(\phi)$ is $\mathcal{L}$-valid.

\subsection{Satisfiability-preserving map}

Let us define the mapping $t$ from the set of $\mathcal{L}$-formulae into the set of $\mathcal{L}_{d}^{\mathrm{U}}$-formulae where $\mathcal{L}_{d}$ is the standard modal logic from $\mathcal{L}$-see Definition 2.2. For any $\mathcal{L}$-formula $\phi, t(\phi)$ is obtained from $\mathrm{N}_{2}(\phi)$ by the following replacements of the basic modal expressions: $r\left(\delta_{1} \cap-\delta_{1}\right)$ is replaced by $\mathrm{U}$ and $r\left(\alpha_{k}^{*}\right)$ is replaced by $\mathrm{c}_{k}$ for $k \in\left\{0, \ldots, 2^{n}-1\right\}$ where $n=\operatorname{card}\left(\mathrm{P}_{0}(\phi)\right)$.

Example 3.1. Let $\mathcal{L}$ be the Rare-logic defined in Example 2.1 and $\phi$ be the $\mathcal{L}$-formula:

$$
\left[r\left(\delta_{1}\right)\right] \mathrm{p} \wedge\left[r\left(\delta_{1} \cap \delta_{2}\right)\right] \mathrm{q} \wedge\left[r\left(\delta_{1} \cap \delta_{2} \cap-\delta_{1}\right) \cup^{*} r\left(\delta_{2}\right)\right] \mathrm{p}
$$

By definition, $t(\phi)=\left[\mathrm{c}_{0} \cap \mathrm{c}_{2}\right] \mathrm{p} \wedge\left[\mathrm{c}_{0}\right] \mathrm{q} \wedge\left[\mathrm{U} \cup^{*}\left(\mathrm{c}_{0} \cap \mathrm{c}_{1}\right)\right] \mathrm{p}$.

Proposition 3.3 below states that $t$ is a satisfiability-preserving transformation from $\mathcal{L}$-satisfiability into $\mathcal{L}_{d}^{\mathrm{U}}$-satisfiability.

Proposition 3.3. [DG00a, Part I] $\phi$ is $\mathcal{L}$-satisfiable iff $t(\phi)$ is $\mathcal{L}_{d^{-}}^{\mathrm{U}}$ satisfiable.

The proof of Proposition 3.3 uses the semilattice structure of the family of relations of the models.

Proof: First assume that $\phi$ is $\mathcal{L}$-satisfiable. So there exist an $\mathcal{L}$-model

$$
\left\langle W, P A R,\left(\mathcal{R}_{P}\right)_{P \subseteq P A R}, V\right\rangle
$$


and $w \in W$ such that $\mathcal{M}, w \models \phi$ and therefore $\mathcal{M}, w \models \mathbb{N}_{2}(\phi)$. The structure $\left\langle\left\{\mathcal{R}_{P}: P \subseteq P A R\right\}, \sqcap, W^{2}\right\rangle$ and the map $V$ satisfy the hypothesis of Proposition 3.1 with the set of parameter constants occurring in $\phi$. If such a set is empty, $\phi$ is a formula of the classical propositional calculus and therefore the proposition trivially holds. Otherwise, by Proposition 3.1, there is a family $\left(Y_{\mathrm{C}}\right)_{\mathrm{c} \in \mathrm{M}_{0}}$ satisfying the conditions $(\mathrm{C} 1)$ and $(\mathrm{C} 2)$ from Proposition 3.1. Let $\mathcal{M}^{\prime}$ be the $\mathcal{L}_{d}^{\mathrm{U}}$-model $\left\langle W,\left(R_{\mathrm{a}}\right)_{\mathrm{a} \in \mathrm{M}}, V^{\prime}\right\rangle$ such that,

- for any $\mathrm{p} \in \mathrm{For}_{0}, V^{\prime}(\mathrm{p}) \stackrel{\text { def }}{=} V(\mathrm{p})$;

- for any $\mathrm{c} \in \mathrm{M}_{0} R_{\mathrm{C}} \stackrel{\text { def }}{=} Y_{\mathrm{C}}$;

- $R_{\oplus\left(\mathrm{a}_{1}, \ldots, \mathrm{a}_{s}\right)} \stackrel{\text { def }}{=} \mathcal{I}(\oplus)(W)\left(R_{\mathrm{a}_{1}}, \ldots, R_{\mathrm{a}_{s}}\right)$ for $\oplus\left(\mathrm{a}_{1}, \ldots, \mathrm{a}_{s}\right) \in \mathrm{M}$ (of $\left.\mathcal{L}_{d}^{\mathrm{U}}\right)$.

It is a routine task to check that $\mathcal{M}^{\prime}$ is an $\mathcal{L}_{d}^{\mathrm{U}}$-model. Furthermore, for any $\alpha \in \mathrm{P}$ occurring in $\phi$ such that $\alpha \equiv \alpha_{i_{1}}^{*} \cup \ldots \cup \alpha_{i_{l}}^{*}, \mathcal{R}_{V(\alpha)}=$ $R_{\mathrm{C}_{i_{1}} \oplus_{\mathcal{L}} \ldots \oplus_{\mathcal{L}} \mathrm{c}_{i_{l}}}=\mathcal{R}_{V\left(\mathrm{~N}_{2}(\alpha)\right)}$ and for any $\alpha \in$ P such that $\alpha \equiv \perp, \mathcal{R}_{V(\alpha)}=$ $R_{\mathrm{U}}=W \times W$. So $\mathcal{M}^{\prime}, w=t(\phi)$.

Now assume that $t(\phi)$ is $\mathcal{L}_{d}^{\mathrm{U}}$-satisfiable. So there exist an $\mathcal{L}_{d}^{\mathrm{U}}$-model $\mathcal{M}=\left\langle W,\left(R_{\mathrm{a}}\right)_{\mathrm{a} \in \mathbb{M}}, V\right\rangle$ and $w \in W$ such that $\mathcal{M}, w \models t(\phi)$. The restriction of $\left(R_{\mathrm{a}}\right)_{\mathrm{a} \in \mathrm{M}}$ to $\mathrm{M}_{0}$, say $\left(R_{\mathrm{C}}\right)_{\mathrm{c} \in \mathrm{M}_{0}}$, satisfies the conditions (H1) and (H2) from Proposition 3.2. By Proposition 3.2, there is a structure $\left\langle\left\{\mathcal{R}_{P}: P \subseteq P A R\right\}, \sqcap, W^{2}\right\rangle$ and a P-valuation $V^{\prime \prime}: \mathrm{P} \rightarrow \mathcal{P}(P A R)$ such that

1. $\left\langle\left\{\mathcal{R}_{P}: P \subseteq P A R\right\}, \sqcap, W^{2}\right\rangle$ is a semilattice with zero element $W^{2}$

2. $\mathcal{R}_{\emptyset}=W^{2}$ and for any $P, P^{\prime} \subseteq P A R, \mathcal{R}_{P \cup P^{\prime}}=\mathcal{R}_{P} \sqcap \mathcal{R}_{P^{\prime}}$;

3. $\operatorname{card}(P A R)=2^{n}$.

Let $\mathcal{M}^{\prime} \stackrel{\text { def }}{=}\left\langle W, P A R,\left(\mathcal{R}_{P}\right)_{P \subseteq P A R}, V^{\prime}\right\rangle$ be the $\mathcal{L}$-model such that

- $V^{\prime}$ restricted to $\mathrm{P}$ is the restriction of $V^{\prime \prime}$;

- for any $\mathrm{p} \in$ For $_{0}, V^{\prime}(\mathrm{p}) \stackrel{\text { def }}{=} V(\mathrm{p})$;

- $V^{\prime}\left(\oplus\left(\mathrm{a}_{1}, \ldots, \mathrm{a}_{s}\right)\right) \stackrel{\text { def }}{=} \mathcal{I}(\oplus)(W)\left(V^{\prime}\left(\mathrm{a}_{1}\right), \ldots, V^{\prime}\left(\mathrm{a}_{s}\right)\right)$ for any $\oplus\left(a_{1}, \ldots, a_{s}\right) \in M($ of $\mathcal{L})$.

It is easy to check that $\mathcal{M}^{\prime}$ is an $\mathcal{L}$-model. Additionnally, for any $\alpha \in \mathrm{P}$ occurring in $\phi$ such that $\alpha \equiv \alpha_{i_{1}}^{*} \cup \ldots \cup \alpha_{i_{l}}^{*}, \mathcal{R}_{V(\alpha)}=$ 
$R_{\mathrm{C}_{i_{1}} \oplus \mathcal{L} \ldots \oplus \mathcal{L} \mathrm{C}_{i_{l}}}$ and for any $\alpha \in \mathrm{P}$ such that $\alpha \equiv \perp, \mathcal{R}_{V(\alpha)}=R_{\mathrm{U}}$. So $\mathcal{M}^{\prime}, w \models \mathrm{N}_{2}(\phi)$. Q.E.D.

Proposition 3.3 entails that $\mathcal{L}_{d}^{\mathrm{U}}$ is decidable only if $\mathcal{L}$ is decidable. Proposition 3.4 will help stating the converse.

Proposition 3.4. There exists a polynomial-time many-one reduction (see e.g. [Pap94]) from $\mathcal{L}_{d}^{U}$-satisfiability into $\mathcal{L}$-satisfiability.

The idea of the proof of Proposition 3.4 consists in defining a reverse map of $t$.

Using the construction of the proof of Proposition 3.3, one can prove the proposition below.

Corollary 3.5. Let $\mathcal{L}$ be a Rare-logic from the class considered so far.

1. $\mathcal{L}$-satisfiability is decidable iff $\mathcal{L}_{d}^{U}$-satisfiability is decidable.

2. $\mathcal{L}$ has the finite model property iff $\mathcal{L}_{d}^{\mathrm{U}}$ has the finite model property.

3. Any $\mathcal{L}$-satisfiable formula $\phi$ has an $\mathcal{L}$-model such that $\operatorname{card}(P A R) \leq$ $2^{|\phi|}$.

4. If $\mathcal{L}_{d}^{\mathrm{U}}$ is U-simplifiable, then $\mathcal{L}$-satisfiability is decidable iff $\mathcal{L}_{d}^{\mathrm{U}-}$ is decidable and $\mathcal{L}$ has the finite model property iff $\mathcal{L}_{d}^{\mathrm{U}-}$ has the finite model property.

Example 3.2. Let $\mathcal{L}$ be the Rare-logic $\langle\mathrm{L}, \mathcal{I}, \mathcal{C}, X\rangle$ such that $O P=$ $\left\{\cap, \circ,{ }^{*}, \cup,{ }^{-1}\right\}, X=F r^{2}$ and $\cap\left[\right.$ resp. $\left.\circ,{ }^{*}, \cup,{ }^{-1}\right]$ is interpreted as the intersection [resp. composition, Kleene star, union, converse]. PDL with the operators $\circ,{ }^{*}, \cup,{ }^{-1}, \cap$ (and without the test operator '?') is a fragment of $\mathcal{L}_{d}^{\mathrm{U}}$ and does not have the finite model property -see e.g. [Vak92]. By Corollary 3.5, $\mathcal{L}$ does not have the finite model property.

The flexibility of the translations allows an extension when nominals are included in the language (see e.g. [DG00a, Part II]) although it is technically more involved. So when Rare-logics can be translated into well-known modal logics, we may obtain straightforward results about the Rare-logics (decidability and possibly complexity upper bounds, ...). 


\section{Decidability}

\subsection{A logic of indiscernibility relations.}

Indiscernibility (see Section 2.3) is a central concept to study relationships between objects in information systems [Paw81]. The logic of indiscernibility relation LIR has been introduced in [Orło93] and it has been defined in Example 2.1.

Proposition 4.1. The LIR-satisfiability problem is decidable iff the DAL-satisfiability problem is decidable.

Proof:(sketch) Since $\mathrm{LIR}_{d}^{\mathrm{U}}$ is U-simplifiable, by Corollary 3.5, LIR is decidable iff $\mathrm{LIR}_{d}^{\mathrm{U}-}$ is decidable. $\mathrm{LIR}_{d}^{\mathrm{U}-}$ is exactly the logic DAL defined in $[\mathrm{FdCO} 85]$ to which is added the universal modal connective $[\mathrm{U}]$. One can show that DAL (or equivalently $\mathrm{LIR}_{d}$ ) is decidable iff $\mathrm{LIR}_{d}^{\mathrm{U}-}$ is decidable. Indeed, for any formulae $\phi, \psi, \phi=_{L I R_{d}} \psi$ iff $[\mathrm{a}] \phi \Rightarrow \psi$ is $\operatorname{LIR}_{d^{-}}$valid with a $\stackrel{\text { def }}{=} \mathrm{c}_{1} \cup^{*} \ldots \cup^{*} \mathrm{c}_{n}$ and $\left\{\mathrm{c}_{1}, \ldots, \mathrm{c}_{n}\right\}$ is the set of modal constants occurring in $\{\phi, \psi\}$. The other direction is shown in [DG00a, Part II] by using some techniques from [GP92].

\section{Q.E.D.}

Decidability of DAL is open although various attempts to prove such a result can be found in the literature (see e.g. [AT89]). This fact is rather surprising considering that after all, DAL is similar to various other polymodal logics, among them the Propositional Dynamic Logic. It is not difficult to show that if PDL with converse and intersection is decidable (which is commonly conjectured in the literature) then DAL is also decidable. By contrast, the logic "LIR without $U^{*}$ ", say $\mathrm{LIR}^{\prime}$, is known to be decidable. Indeed, LIR' is decidable iff $\mathrm{LIR}_{d}^{\prime \mathrm{U}_{-}}$is decidable. However, $\mathrm{LIR}_{d}^{\prime \mathrm{U}_{-}}$is decidable (see e.g. [Dem99]). Consequently, the logic of indiscernibility relations defined in [Orło84b] is decidable.

\subsection{A logic with knowledge operators.}

The logic with knowledge operators LKO has been introduced in [Orło89] to model reasoning in presence of incomplete information. The ontology from which are defined the semantical structures of LKO is alternative to the usual Kripke-style semantics. Indeed, the worlds in a model are not interpreted as knowledge states but as objects. 
Similarly, the relations are not interpreted as compatibility relations but as indiscernibility relations between objects with respect to sets of agents. The set of formulae of LKO is the smallest set that contains For $_{0}$ and it is closed under $\neg, \wedge$ and under the unary operators from $\{K(\alpha): \alpha \in \mathrm{P}\}$ where $\mathrm{P}$ is a set of parameter expressions. An LKO-model

$$
\mathcal{M}=\left\langle O B, A G T,\left(R_{Q}\right)_{Q \subseteq A G T}, V\right\rangle
$$

is a structure such that:

1. $O B$ is a non-empty set of objects; $A G T$ is a non-empty set of agents;

2. for any $Q, Q^{\prime} \subseteq A G T, R_{Q}$ is an equivalence relation, $R_{Q \cup Q^{\prime}}=$ $R_{Q} \cap R_{Q^{\prime}}$ and $R_{\emptyset}=O B \times O B$;

3. $V$ is a mapping For $_{0} \cup \mathrm{P} \rightarrow \mathcal{P}(O B) \cup \mathcal{P}(A G T)$ such that $V(\mathrm{p}) \subseteq$ $O B$ for any $\mathrm{p} \in \mathrm{For}_{0}$ and $V$ restricted to $\mathrm{P}$ is a $\mathrm{P}$-valuation.

The satisfiability relation $=$ is defined as usual, except for the following condition: $\mathcal{M}, w=K(\alpha) \phi \stackrel{\text { def }}{\Leftrightarrow}$ either for any $w^{\prime} \in R_{V(\alpha)}(w), \mathcal{M}, w^{\prime}=$ $\phi$ or for any $w^{\prime} \in R_{V(\alpha)}(w), \mathcal{M}, w^{\prime} \models \neg \phi . K(\alpha) \phi$ can be interpreted by: the set $\alpha$ of agents knows whether $\phi$ holds (see e.g. [Orło89]). The notion of LKO-validity, LKO-satisfiability, ..., are defined in the standard way. Let $g$ be the mapping from the set of LKO-formulae into the set of $\mathrm{LIR}^{\prime}$-formulae such that

- $g(\mathrm{p}) \stackrel{\text { def }}{=} \mathrm{p}$ for any $\mathrm{p} \in$ For $_{0} ; g(\neg \phi) \stackrel{\text { def }}{=} \neg g(\phi)$;

- $g(\phi \wedge \psi) \stackrel{\text { def }}{=} g(\phi) \wedge g(\psi) ; g(K(\alpha) \phi) \stackrel{\text { def }}{=}[r(\alpha)] g(\phi) \vee[r(\alpha)] g(\neg \phi)$.

Proposition 4.2. For any LKO-formula $\phi, \phi$ has an LKO-model of the form $\langle O B, A G T, \ldots\rangle$ iff $g(\phi)$ has an $\mathrm{LIR}^{\prime}$-model of the form $\langle O B, A G T, \ldots\rangle$.

As a corollary, LKO-satisfiability problem is decidable. Moreover, it is possible to define a polynomial-time many-one reduction from

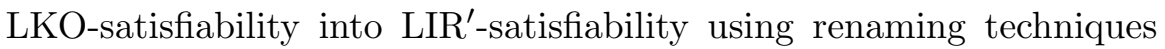
(see e.g. [Min88]).

\subsection{Modal logics with a fixed set of parameters}

The language $\mathrm{L}$ of the modal logics for parameters contains a fixed countable set PAR of parameters. The modal expressions of the language are the subsets of PAR. Hence, the modal operators are indexed 
by sets of parameters. Such logics have been considered for instance in [Bal96b, Bal97]. To be precise, we shall not deal with sets but rather with a finite representation of certain sets. This representational aspect shall be emphasize when needed but this is really necessary since we wish to establish decidability results. For example, we want to be able to decide whether two sets that occur in a formula are equal, which seems to be a reasonable requirement.

By an L-frame (for such kinds of logics) we understand a pair $\left\langle W,\left(R_{P}\right)_{P \subseteq \mathrm{PAR}}\right\rangle$ such that $W$ is a non-empty set and for any $P \subseteq \mathrm{PAR}$, $R_{P}$ is a binary relation on $W$. By an L-model $\mathcal{M}$, we understand a triple $\left\langle W,\left(R_{P}\right)_{P \subseteq \mathrm{PAR}}, V\right\rangle$ such that $\mathcal{F}=\left\langle W,\left(R_{P}\right)_{P \subseteq \mathrm{PAR}}\right\rangle$ is an Lframe and $V$ is a mapping For $_{0} \rightarrow \mathcal{P}(W)$. The satisfiability relation is defined in the usual way. In this paper, by a modal logic for parameters $\mathcal{L}$, we understand (this is a restricted sense in comparison with the notion introduced in [Bal97, DG00a]) a triple $\langle\mathrm{L}, X, \mathcal{C}\rangle$ such that

- L is a language for modal logics for parameters;

- $X$ is a class of modal frames;

- $\mathcal{C}$ is the class of L-models such that for any $\mathcal{M}=\left\langle W,\left(R_{P}\right)_{P \subseteq \mathrm{PAR}}, V\right\rangle$,

1. $R_{\emptyset}=W^{2}$ and for any $P, P^{\prime} \subseteq \mathrm{PAR}, R_{P \cup P^{\prime}}=R_{P} \cap R_{P^{\prime}}$;

2. for any $\emptyset \neq P \subseteq$ PAR, $\left\langle W, R_{P}\right\rangle \in X$.

The notion of $\mathcal{L}$-satisfiability, $\mathcal{L}$-validity, logical $\mathcal{L}$-consequence etc, ... are defined in the usual way. Let $\mathcal{L}$ be a modal logic for parameters $\langle\mathrm{L}, X, \mathcal{C}\rangle$. The Rare-logic $\operatorname{RARE}(\mathcal{L})=\left\langle\mathrm{L}^{\prime}, \mathcal{I}^{\prime}, \mathcal{C}^{\prime}, X^{\prime}\right\rangle$ is called the Rarelogic from $\mathcal{L} \stackrel{\text { def }}{\Leftrightarrow}$

- $\mathrm{L}^{\prime}$ is a language for Rare-logic such that $O P \stackrel{\text { def }}{=}\{\cap\}$ and $\mathcal{I}$ interprets $\cap$ as set intersection;

- $X^{\prime} \stackrel{\text { def }}{=} X$

- $\mathcal{C}^{\prime}$ is the unique set of $\mathrm{L}^{\prime}$-models respecting $\mathcal{I}^{\prime}$ making $\operatorname{RARE}(\mathcal{L})$ a Rare-logic of the class considered so far.

Let $\mathcal{L}$ be a modal logic for parameters. Let $\phi$ be an L-formula such that the only sets occurring in $\phi$ are $X_{1}, \ldots, X_{n}$. For any integer $k \in\left\{0, \ldots, 2^{n}-1\right\}$, we write $X_{k}^{*}$ to denote the set

$$
X_{k}^{*} \stackrel{\text { def }}{=} Y_{1} \cap \ldots \cap Y_{n}
$$


where for any $i \in\{1, \ldots, n\}, Y_{i} \stackrel{\text { def }}{=} X_{i}$ if $b_{i t}(k)=0$ otherwise $Y_{i} \stackrel{\text { def }}{=}$ PAR $\backslash X_{i}$. Hence, $X_{k}^{*}$ is a concrete set (not a Boolean expression as done until now). The set $\left\{X_{k}^{*}: k \in\left\{0, \ldots, 2^{n}-1\right\}, X_{k}^{*} \neq \emptyset\right\}$ is a partition of PAR. For each set $X_{i}$ we associate a parameter constant $\delta_{i}$. For any integer $k \in\left\{0, \ldots, 2^{n}-1\right\}$, we write $\alpha_{k}^{*}$ to denote the Boolean expression $\alpha_{k}^{*} \stackrel{\text { def }}{=} \alpha_{1} \cap \ldots \cap \alpha_{n}$ where for any $i \in\{1, \ldots, n\}$, $\alpha_{i} \stackrel{\text { def }}{=} \delta_{i}$ if $b_{i t}(k)=0$ otherwise $\alpha_{i} \stackrel{\text { def }}{=}-\delta_{i}$. We write $t(\phi)$ to denote the $\operatorname{RARE}(\mathcal{L})$-formula obtained from $\phi$ by substituting $X_{i} \neq \emptyset$ by $t\left(X_{i}\right)$ defined below by

$$
t\left(X_{i}\right) \stackrel{\text { def }}{=} r\left(\bigcup\left\{\alpha_{k}^{*}: k \in\left\{0, \ldots, 2^{n}-1\right\}, X_{k}^{*} \neq \emptyset, b i t_{i}(k)=0\right\}\right)
$$

In the case when $X_{i}=\emptyset, \emptyset$ is substituted by $r\left(\delta_{1} \cap-\delta_{1}\right)$. It is worth noting that since we have not yet fixed the mode of representation of the sets $P \subseteq$ PAR, it might not be decidable to know whether $X_{k}^{*}=\emptyset$ or $X_{i}=\emptyset$. But, we can show that

Proposition 4.3. $\phi$ is $\mathcal{L}$-satisfiable iff $t(\phi)$ is $\operatorname{RARE}(\mathcal{L})$-satisfiable.

Proof: Let $\mathcal{M}=\left\langle W,\left(R_{P}\right)_{P \subset \mathrm{PAR}}, V\right\rangle$ be an $\mathcal{L}$-model and $w \in W$ such that $\mathcal{M}, w=\phi$. Consider the $\operatorname{RARE}(\mathcal{L})$-model $\mathcal{M}^{\prime}=\left\langle W, \operatorname{PAR},\left(\mathcal{R}_{P}\right)_{P \subseteq \mathrm{PAR}}, V^{\prime}\right\rangle$ such that

- for any $P \subseteq$ PAR, $\mathcal{R}_{P} \stackrel{\text { def }}{=} R_{P}$;

- the restriction of $V^{\prime}$ to the set of propositional variables is $V$;

- for any $j \in\{1, \ldots, n\}, V^{\prime}\left(\delta_{j}\right) \stackrel{\text { def }}{=} X_{j}$.

Hence for any $j \in\{1, \ldots, n\}, V^{\prime}\left(t\left(X_{j}\right)\right)=X_{j}$. It is a routine task to check that $\mathcal{M}^{\prime}, w \models t(\phi)$ and $\mathcal{M}^{\prime}$ is a $\operatorname{RARE}(\mathcal{L})$-model.

Now let $\mathcal{M}^{\prime}=\left\langle W, P A R,\left(\mathcal{R}_{P}\right)_{P \subseteq P A R}, V^{\prime}\right\rangle$ be a $\operatorname{RARE}(\mathcal{L})$-model and $w \in W$ be such that $\mathcal{M}^{\prime}, w \models t(\phi)$. The set $P A R$ does not have to be equal to PAR (fixed for $\mathcal{L}$ ). Consider the $\mathcal{L}$-model $\mathcal{M}=$ $\left(W,\left(R_{P}\right)_{P \subseteq \mathrm{PAR}}, V\right)$ such that

- $V$ is the restriction of $V^{\prime}$ to the set of propositional variables;

- for any $k \in\left\{0, \ldots, 2^{n}-1\right\}$ and $x \in X_{k}^{*}, R_{\{x\}} \stackrel{\text { def }}{=} \mathcal{R}_{V^{\prime}\left(\alpha_{k}^{*}\right)}$ and $R_{\emptyset} \stackrel{\text { def }}{=} W \times W$

- for any $\emptyset \neq P \subseteq$ PAR, we write $\left\{X_{i_{1}}^{*}, \ldots, X_{i_{l}}^{*}\right\}$ to denote the smallest set of non-empty sets (with respect to set inclusion) 
such that $P \subseteq X_{i_{1}}^{*} \cup \ldots \cup X_{i_{l}}^{*} .\left\{X_{i_{1}}^{*}, \ldots, X_{i_{l}}^{*}\right\}$ always exists and is unique. We define $R_{P}$ as follows

$$
R_{P} \stackrel{\text { def }}{=} \bigcap\left\{R_{\{x\}}: x \in X_{i_{u}}^{*}, u \in\{1, \ldots, l\}\right\}
$$

It is a routine task to check that the definition is correct. We can also show that for any $P, P^{\prime} \subseteq$ PAR $R_{P \cup P^{\prime}}=R_{P} \cap R_{P^{\prime}}$. It remains to prove that $R_{X_{k^{\prime}}}=\mathcal{R}_{V^{\prime}\left(t\left(X_{k^{\prime}}\right)\right)}$ for $k^{\prime} \in\{1, \ldots, n\}$. First, for any $j \in\left\{0, \ldots, 2^{n}-1\right\}$, if $X_{j}^{*} \neq \emptyset$, then $R_{X_{j}^{*}}=\mathcal{R}_{V^{\prime}\left(\alpha_{j}^{*}\right)}$.

$$
R_{X_{j}}=R_{\bigcup\left\{X_{k}^{*}: k \in\left\{0, \ldots, 2^{n}-1\right\}, b i t_{j}(k)=0, X_{k}^{*} \neq \emptyset\right\}}
$$

(by definition of the $X_{k}^{*}$ 's)

$$
\begin{aligned}
& =\bigcap\left\{R_{X_{k}^{*}}: k \in\left\{0, \ldots, 2^{n}-1\right\}, b i t_{j}(k)=0, X_{k}^{*} \neq \emptyset\right\} \\
& =\bigcap\left\{R_{V^{\prime}\left(\alpha_{k}^{*}\right)}: k \in\left\{0, \ldots, 2^{n}-1\right\}, \text { bit }_{j}(k)=0, X_{k}^{*} \neq \emptyset\right\} \\
& \text { (see the preliminary remark) } \\
& =\mathcal{R}_{V^{\prime}\left(\cup\left\{\alpha_{k}^{*}: k \in\left\{0, \ldots, 2^{n}-1\right\}, b i t_{j}(k)=0, X_{k}^{*} \neq \emptyset\right\}\right)} \\
& \left(\mathcal{M}^{\prime} \text { is a } \operatorname{RARE}(\mathcal{L}) \text {-model }\right) \\
& =\mathcal{R}_{V^{\prime}\left(t\left(X_{j}^{*}\right)\right)}
\end{aligned}
$$

Q.E.D.

Corollary 4.4. Let $\mathcal{L}$ be a modal logic for parameters. Let $Z$ be a class of $\mathcal{L}$-formulae such that for any $\phi \in Z$ it is decidable whether (see the notations above) (D1) $X_{i}$ is empty where $X_{1}, \ldots, X_{n}$ are representations of the sets occurring in $\phi$ and (D2) $X_{k}^{*}$ is empty $(0 \leq$ $\left.k \leq 2^{n}-1\right)$. Then, if the corresponding Rare-logic $\operatorname{RARE}(\mathcal{L})$ has a decidable satisfiability problem, then the $\mathcal{L}$-satisfiability problem for the fragment $Z$ is decidable.

Let PAR $=\left\{p_{1}, p_{2}, \ldots\right\}$ be a countable set of parameters (not necessarily finite). A natural representation of the finite subset $\left\{p_{1}, \ldots, p_{k}\right\}$ of PAR is $\left\{{ }^{f} p_{1}, \ldots, p_{k}\right\}^{f} . '\left\{{ }^{f},{ }^{\prime},\right\}^{f}$ ' and ',' are symbols of the language. Each cofinite subset PAR $\backslash\left\{p_{1}, \ldots, p_{k}\right\}$ can be represented by $\left\{{ }^{c} p_{1}, \ldots, p_{k}\right\}^{c}$. ${ }^{\prime}\left\{{ }^{c} \text { ' and ' }\right\}^{c}$ ' are symbols of the language. Let $Z^{f c}$ be the set of $\mathcal{L}$ formulae such that only finite or cofinite sets of parameters occur and the representation above is used. Then $Z^{f c}$ satisfies the hypothesis of Corollary 4.4. Moreover, if the representation of a set $Y$ occurs in a formula, then the representation of the set PAR $\backslash Y$ can also occur in a formula of $Z^{f c}$. Similar classes of formulae have been considered for instance in [Bal97].

By way of example we can state that 
Proposition 4.5. Let $\mathcal{L}=\langle\mathrm{L}, X, \mathcal{C}\rangle$ be a modal logic for parameters. $\mathcal{L}$-satisfiability restricted to some $Z$ satisfying (D1) and (D2) in Corollary 4.4 is decidable when $X$ is either the set of transitive frames or the set of reflexive and transitive frames.

Other decidability results for logics with relative accessibility relations (involving nominals) can be found in [DK98].

\section{Complexity}

The map $t$ defined in Section 3 may increase exponentially the size of formulae although the number of subformulae does not change. In this section, we provide a sufficient condition so that $\mathcal{L}$-satisfiability is in EXPTIME. That is, it can be solved by a deterministic Turing machine in exponential-time (see e.g. [Pap94]).

Lemma 5.1. Let $\phi$ be a formula.

1. $t(\phi)$ can be computed in deterministic time $\mathcal{O}\left(2^{p_{1}(|\phi|)}\right)$ for some polynomial $p_{1}($.$) ;$

2. $\operatorname{card}(\operatorname{sub}(\phi))=\operatorname{card}(\operatorname{sub}(t(\phi)))$;

3. $|t(\phi)|$ is in $\mathcal{O}\left(2^{p_{2}(|\phi|)}\right)$ for some polynomial $p_{2}($.$) ;$

The proof is by simple inspection of the definition of $t$.

Proposition 5.2. Let $\mathcal{L}$ be a Rare-logic of the kind considered so far.

1. There is a polynomial-time many-one reduction from $\mathcal{L}_{d}^{U}$-satisfiability into $\mathcal{L}$-satisfiability.

2. If the $\mathcal{L}_{d}^{\mathrm{U}}$-satisfiability can be solved by a deterministic Turing machine in time $\mathcal{O}\left(q_{1}(|\phi|)+2^{q_{2}(\operatorname{card}(\operatorname{sub}(\phi)))}\right)$ for some polynomials $q_{1}($.$) and q_{2}($.$) , then \mathcal{L}$-satisfiability is in EXPTIME.

Proof: (1) See Proposition 3.4. (2) We know that $\phi$ is $\mathcal{L}$-satisfiable iff $t(\phi)$ is $\mathcal{L}_{d}^{\mathrm{U}}$-satisfiable. By using Lemma 5.1, deciding whether $\phi$ is $\mathcal{L}$-satisfiable requires time in

$$
\mathcal{O}\left(2^{p_{1}(|\phi|)}+q_{1}\left(2^{p_{2}(|\phi|)}\right)+2^{q_{2}(\operatorname{card}(\operatorname{sub}(\phi)))}\right)
$$

which is also in time $\mathcal{O}\left(2^{p(|\phi|)}\right)$ for some polynomial $p($.$) . Q.E.D.$ 
Corollary 5.3. [Dem99] LIR'-satisfiability and LKO-satisfiability are EXPTIME-complete.

To establish the complexity lower bounds, general results from [Hem96] are used.

\section{Proof systems for LKO}

Proposition 3.3 is not only meaningful to prove decidability or the finite model property but it also helps to define proof systems for Rare-logics from proof systems for the corresponding standard modal logics. In the sequel, we concentrate on the logic LKO that can be equipped with Hilbert-style proof system [Dem99]. Let $\mathcal{L}$ be a Rare-logic of the class studied so far and $\mathcal{L}_{d}^{\mathrm{U}}$ be the corresponding standard modal logic. Assume that $\mathcal{L}_{d}^{\mathrm{U}}$ is equipped with a proof system $\vdash^{*}$. A proof system $\vdash$ for $\mathcal{L}$ should first be able to simulate the normalization process. For instance, the following equivalences have to be encoded:

1. $[r(\alpha)] \mathrm{p} \Leftrightarrow[r(\beta)] \mathrm{p}$ when $\alpha \equiv \beta$;

2. $[r(\alpha \cup \beta)] \mathrm{p} \Leftrightarrow\left[r(\alpha) \oplus_{\mathcal{L}} r(\beta)\right] \mathrm{p}$.

For instance 1. and 2. above can be viewed as axiom schemes of Hilbert-style systems. The rest of the calculus $\vdash$ consists of $\vdash^{*}$ where only normalized formulae are admitted and the basic modal expressions of the form $r(\delta \cap-\delta)$ should play the role of U. By normalized formula, we mean a formula $\phi$ such that $\mathrm{N}_{2}(\phi)=\phi$ modulo associativity and commutativity of $\cap$ and $\cup$. Then, in $\vdash$ the basic modal expressions of the form $r\left(\alpha_{k}^{*}\right)$ play the role of the modal constants $\mathrm{c}_{k}$ in $\vdash^{*}$. Although described at some informal level, this program can be easily implemented for particular logics and proof systems. This was first done in [Kon97] for logics with relative similarity relations. This has been more systematically pursued for Hilbert-style proof systems in [DG00a, Part II]. By way of example, the Hilbert-style proof system $\vdash_{l k o}$ for the logic LKO is composed of the axiom schemes

- the tautologies of the Propositional Calculus;

- $K(\alpha) \mathrm{p} \Rightarrow K\left(\alpha^{\prime}\right) \mathrm{p}$ when $\alpha \sqsubseteq \alpha^{\prime} ; \phi \wedge K(\alpha) \mathrm{p} \wedge K(\alpha)(\mathrm{p} \Rightarrow \mathrm{q}) \Rightarrow$ $K(\alpha) \mathrm{q}$

- $K(\alpha)(K(\alpha) \mathrm{p} \Rightarrow \mathrm{p}) ; K(\alpha) \mathrm{p} \Leftrightarrow K(\alpha) \neg \mathrm{p}$. 
and of the inference rules

1. modus ponens: $\frac{\phi \phi \Rightarrow \psi}{\psi}$;

2. necessitation: $\frac{\phi}{K(\alpha) \phi}$ for any $\alpha \in \mathrm{P}$.

Proposition 6.1. [Dem99] $\vdash_{l k o} \phi$ iff $\phi$ is LKO-valid.

Display Logic (DL) is a proof-theoretical framework introduced in [Bel82] that admits a very general cut-elimination theorem. Moreover, DL generalises the structural language of Gentzen's sequents in a rather abstract way by using multiple complex structural connectives instead of Gentzen's comma. A display logic calculus for LKO can be found in [DG00b].

\section{Concluding Remarks}

In this paper, we have presented many-one reductions between satisfiability problems for Rare-logics and standard modal logics. Both directions have been investigated and in some sense, we have seen that the Rare-logics can be very similar to standard modal logics. The present short survey has been the opportunity to present results for particular classes of Rare-logics (because of lack of space the presentation of other classes had to be skipped but most of them can be found in [DG00a]). When the standard modal logics corresponding to the Rare-logics are well-known, we get serious insights about the Rare-logics (decidability, axiomatization, complexity upper bounds, ...). Solving the following open questions will help solving similar questions for Rare-logics thanks to the translation presented here:

1. Axiomatization and decidability status of DAL (if PDL + converse and intersection is decidable then DAL is decidable);

2. Decidability of Combinatory PDL (CPDL) [PT91] with converse and intersection.

\section{References}

[AT89] D. Archangelsky and M. Taitslin. A logic for data description. In A. Meyer and M. Taitslin, editors, Symposium on Logic Foundations of Computer Science, Pereslavl-Zalessky, pages 2-11. 
Springer-Verlag, Lecture Notes in Computer Science, Vol. 363, July 1989.

[Bal96a] Ph. Balbiani. A modal logic for data analysis. In W. Penczek and A. Szalas, editors, 21st Symposium on Mathematical Foundations of Computer Sciences (MFCS'96), Krakow, pages 167-179. Lecture Notes in Computer Science, Vol. 1113, Springer-Verlag, 1996.

[Bal96b] Ph. Balbiani. Modal logics with relative accessibility relations. In D. Gabbay and H.J. Ohlbach, editors, Conference on Formal and Applied Practical Reasoning (FAPR'96), Bonn, pages 29-41. Springer-Verlag, June 1996.

[Bal97] Ph. Balbiani. Axiomatization of logics based on Kripke models with relative accessibility relations. In [Oe97], pages 553-578, 1997.

[Bel82] N. Belnap. Display logic. J. of Philosophical Logic, 11:375-417, 1982.

[BO99] Ph. Balbiani and E. Orlowska. A hierarchy of modal logics with relative accessibility relations. J. of Applied Non-Classical Logics, special issue in the Memory of George Gargov, 9:303-328, 1999.

[Dem98a] S. Demri. A class of decidable information logics. Theoretical Computer Science, 195(1):33-60, 1998.

[Dem98b] S. Demri. Coping with semilattices of relations in logics with relative accessibility relations (extended abstract). In E. Orłowska and A. Szalas, editors, 4th International Seminar on Relational Methods in Logic, Algebra and Computer Science, pages 43-47, September 1998.

[Dem99] S. Demri. A logic with relative knowledge operators. J. of Logic, Language and Information, 8(2):167-185, 1999.

[DG00a] S. Demri and D. Gabbay. On modal logics characterized by models with relative accessibility relations: Part I and Part II. Studia Logica, 2000. To appear.

[DG00b] S. Demri and R. Goré. Display calculi for logics with relative accessibility relations. J. of Logic, Language and Information, 9(2):213-236, 2000.

[DK98] S. Demri and B. Konikowska. Relative similarity logics are decidable: reduction to $\mathrm{FO}^{2}$ with equality. In JELIA' 98 , pages 279-293. Lecture Notes in Artificial Intelligence, Vol. 1489, Springer-Verlag, 1998. 
[DO99] S. Demri and E. Orłowska. Every finitely reducible logic has the finite model property with respect to the class of $<>$-formulae. Studia Logica, 62(2):177-200, 1999. Special issue edited by M.L. Dalla Chiara and D. Mundici. Selected papers in honour of Ettore Casari.

[Dün97] I. Düntsch. Rough sets and algebras of relations. In [Oe97], pages 95-108, 1997.

[FdCO85] L. Fariñas del Cerro and E. Orłowska. DAL - A logic for data analysis. Theoretical Computer Science, 36:251-264, 1985.

[GP92] V. Goranko and S. Passy. Using the universal modality: gains and questions. J. of Logic and Computation, 2(1):5-30, 1992.

[Har84] D. Harel. Dynamic logic. In D. Gabbay and F. Guenthner, editors, Handbook of Philosophical Logic, Volume II, pages 497-604. Reidel, Dordrecht, 1984.

[Hem96] E. Hemaspaandra. The price of universality. Notre Dame Journal of Formal Logic, 37(2):173-203, 1996.

[Jär97] J. Järvinen. Representation of information systems and dependences spaces, and some basic algorithms. Licentiate's thesis, 1997.

[Kon97] B. Konikowska. A logic for reasoning about relative similarity. Studia Logica, 58(1):185-226, 1997.

[Min88] G. Mints. Gentzen-type and resolution rules part I: propositional logic. In P. Martin-Löf and G. Mints, editors, International Conference on Computer Logic, Tallinn, pages 198-231. Springer Verlag, Lecture Notes in Computer Science, Vol. 417, 1988.

[Nov97] M. Novotny. Applications of dependence spaces. In [Oe97], pages 247-289, 1997.

[Oe97] E. Orłowska (ed.). Incomplete Information: Rough Set Analysis. Studies in Fuzziness and Soft Computing. Physica-Verlag, Heidelberg, 1997.

[Orło84a] E. Orłowska. Logic of indiscernibility relations. In A. Skowron, editor, 5th Symposium on Computation Theory, Zaborów, Poland, pages 177-186. Lecture Notes in Computer Science, Vol. 208, Springer-Verlag, 1984.

[Orło84b] E. Orłowska. Modal logics in the theory of information systems. Zeitschrift für Mathematik Logik und Grundlagen der Mathematik, 30(1):213-222, 1984. 
[Orło88] E. Orłowska. Kripke models with relative accessibility and their applications to inferences from incomplete information. In G. Mirkowska and H. Rasiowa, editors, Mathematical Problems in Computation Theory, pages 329-339. Banach Center Publications, Volume 21 PWN - Polish Scientific Publishers, Warsaw, 1988.

[Orło89] E. Orłowska. Logic for reasoning about knowledge. Zeitschrift für Mathematik Logik und Grundlagen der Mathematik, 35:559-568, 1989.

[Orło93] E. Orłowska. Reasoning with incomplete information: rough set based information logics. In V. Alagar, S. Bergler, and F. Dong, editors, Incompleteness and Uncertainty in Information Systems Workshop, pages 16-33. Springer-Verlag, October 1993.

[Pap94] Ch. Papadimitriou. Computational Complexity. Addison-Wesley Publishing Company, 1994.

[Paw81] Z. Pawlak. Information systems theoretical foundations. Information Systems, 6(3):205-218, 1981.

[PT91] S. Passy and T. Tinchev. An essay in combinatory dynamic logic. Information and Computation, 93:263-332, 1991.

[Tar53] A. Tarski. Undecidable Theories. Studies in Logic and the foundations of Mathematics. North-Holland Publishing Company, 1953. In collaboration with A. Mostowski. and R. Robinson.

[Vak91] D. Vakarelov. Modal logics for knowledge representation systems. Theoretical Computer Science, 90:433-456, 1991.

[Vak92] D. Vakarelov. A modal logic for cyclic repeating. Information and Computation, 101:103-122, 1992. 